\title{
A Comparison Between the Stability Properties in a DDE Model for Leukemia and the Modified Fractional Counterpart
}

\author{
I.R. Rădulescu 1,2,a) , D. Cândea ${ }^{2, b)}$ and E. Kaslik ${ }^{1,3, c)}$ \\ ${ }^{1}$ Institute e-Austria, Timisoara, Romania \\ ${ }^{2}$ Department of Mathematics and Informatics, University Politehnica of Bucharest, Romania \\ ${ }^{3}$ West University of Timisoara, Romania \\ a) Corresponding author: nicola_rodica@yahoo.com \\ b)doinatilivea@gmail.com \\ c)ekaslik@gmail.com
}

\begin{abstract}
In this paper, a delay differential equations (DDEs) model of leukemia is introduced and its dynamical properties are investigated in comparison with the modified fractional-order system where the Caputo's derivative is used. The model takes into account three types of division that a stem-like cell can undergo and cell competition between healthy and leukemia cell populations. The action of the immune system on the leukemic cell populations is also considered. The stability properties of the equilibrium points are established through numerical results and the differences between the two types of approaches are discussed. Medical conclusions are drawn in view of the obtained numerical simulations.
\end{abstract}

\section{INTRODUCTION}

Chronic myeloid leukemia (CML) is a hematopoietic stem cell disorder characterized by the Philadelphia (Ph) chromosome translocation. This translocation causes the transcription of a constitutively active oncogenic enzyme with tyrosine kinase activity: BCR-ABL ([15]). Research in the last decades has ascertained that BCR-ABL is the cause to the CML pathogenesis and that constitutive tyrosine kinase activity is central to BCR-ABL's ability to alter normal hematopoietic cells into leukemic cells ([12]).

Most of the cases are diagnosed during an initial chronic phase characterized by increased proliferation and the accumulation of immature myeloid cells over several years. If untreated, CML inevitably progresses to an accelerated phase and/or blast phase. The treatment of CML has been revolutionized by the advent of specific tyrosine kinase inhibitors (TKI). TKI selectively inhibits BCR-ABL action and, consequently, repress the tyrosine kinase activity. The most important TKI is Imatinib, which has a very good outcome if the treatment is not interrupted ([26]).

It is generally assumed that the immune system plays an essential role in tumor evolution. Researches in cancer immunology imply that both innate and adaptive immunity are implicated in the defense systems against cancer. In CML, leukemic cells express antigens that are immunogenic and can be recognized by cytotoxic T cells (or CD8+ T cells), which are able to eliminate leukemic stem cells (LSCs) ([34]). In [9], the authors claim that some CML patients under imatinib-induced remission develop an anti-leukemia immune response involving both CD4+ and CD8+ T cells. New advances in cancer immunotherapy suggest that the immune system can be used as a tool to prevent or cure cancer [36]. Consequently, immunotherapy should be considered as a complementary therapy, in order to increase the effectiveness of the immune response against cancer. T-cell based therapies have been shown to boost the body's ability to fight cancers as leukemia, lymphoma and breast cancer [13]. Therefore a model that includes T cell dynamics may serve to enhance the potential immunotherapy treatments of CML.

There is a vast literature on mathematical modeling the normal and pathologic hematopoiesis ([1], [6], [10], [11], [17], [18], [32], [33]). In some papers, CML models with competition between cell lines are studied using stochastic differential equations ([7], [31]) while in others ordinary differential equations (ODEs) are used [24], [16], [25], [14]. 
However, because of the complexity of the hematopoietic system, ODEs models cannot always capture the rich variety of dynamics observed in real life. An intermediate approach is the use of delay differential equations (DDEs) models, which exhibit more complex dynamical behaviors (see, for example [2]).

Some models that specifically study the immune response to CML are [21], [5], [28] and [27]. In [21], Kim et al. analyzed a high order DDEs model to account for the role of anti-leukemia specific response in CML dynamics and concluded that the anti-leukemia $T$ cell response may help maintain remission under Imatinib therapy. In [5], the authors analyzed a DDEs model for the dynamics of CML cells and effectors T cells considering Imatinib therapy and immunotherapy. The focus in the paper [28] is on analyzing a DDE model in order to elucidate the transition of leukemia from the stable chronic phase to the unstable accelerated and acute phases. In [27], Moore and Li devise an ODE model and examine which parameters of the model are most important in the success or failure of cancer remission.

The DDEs model we present in Section 2 follows the lines of the models for leukemia developed by Mackey and collaborators ([6], [11], [30]) and is part of a larger study of Halanay and colleagues about CML ([4], [8], [17], [18], [32], [33]). One of the novelties in this study is the consideration of three types of division that a stem-like cell can undergo: asymmetric division, symmetric self-renewal and differentiation ([35]). The DDEs system models the interaction between healthy cell populations, leukemic cell population and active T cells and it consists of a strongly nonlinear five-dimensional system of delay differential equations.

Recently, fractional differential models have been extensively used in different fields, such as physics, biology, chemical technology and economy ([29], [20]). Due to the fact that fractional-order equations are naturally related to systems with memory, which exists in most biological systems, several papers have analyzed the qualitative properties of fractional-order biological models (see e.g. [3]). Based on the reasoning above and because adding a supplementary memory to the DDEs model for CML might be more in line with the real life situation, we then present the fractionalorder DDEs model for CML and realise a comparison between the dynamical properties of the two models.

In the following section, the delay competition model of CML, which was first introduced in [32] (see also [8]), is presented and explained. Based on this model, in Section 3 we introduce the fractional-order DDEs system for CML. Section 4 presents numerical results and simulations, which are then discussed and interpreted from a medical point of view in Section 5.

\section{THE DDE MODEL FOR CML AND EQUILIBRIUM POINTS}

The mathematical model presented in this section consists of a system of strongly nonlinear differential equations with five time lags and five state variables. The time delays represent the duration of the cell cycle $\left(\tau_{1}\right.$ and $\left.\tau_{3}\right)$, the time period needed for differentiation into the leukocyte line $\left(\tau_{2}\right.$ and $\left.\tau_{4}\right)$ and the time period needed for the naive $\mathrm{T}$ cells to finish the minimal developmental program of $n_{1}$ cell divisions $\left(\tau_{5}=n_{1} \tau\right.$, where $\tau$ is duration of one $\mathrm{T}$ cell division). In this model it is assumed that the dynamics of healthy and leukemic cell populations are similar and the only notable difference is in the value of the parameters. Consequently, the subscript of the parameters for the healthy cells is denoted with "h" and the subscript for the abnormal cells is denoted with "l". Also, the third and the fourth equations are similar with the first and the second ones. Three types of division are taken into consideration in this model: asymmetric division $\left(\eta_{1}\right)$, symmetric differentiation $\left(\eta_{2}\right)$ and self-renewal $\left(1-\eta_{1}-\eta_{2}\right)$.

The state variables are five cell populations: healthy stem-like and mature $\left(x_{1}\right.$ and $\left.x_{2}\right)$, leukemic stem-like and mature $\left(x_{3}\right.$ and $\left.x_{4}\right)$ and anti-leukemic T cells involving both CD4+ and CD8+ T cells $\left(x_{5}\right)$. The dynamics of these populations in CML is described by the following system

$$
\begin{aligned}
& \dot{x}_{1}=f_{1}\left(x_{1}, x_{2}, x_{3}, x_{4}, x_{1 \tau_{1}}, x_{2 \tau_{1}}, x_{3 \tau_{1}}, x_{4 \tau_{1}}\right) \\
& \dot{x}_{2}=f_{2}\left(x_{2}, x_{1 \tau_{2}}, x_{2 \tau_{2}}, x_{4 \tau_{2}}\right) \\
& \dot{x}_{3}=f_{3}\left(x_{1}, x_{2}, x_{3}, x_{4}, x_{5}, x_{1 \tau_{3}}, x_{2 \tau_{3}}, x_{3 \tau_{3}}, x_{4 \tau_{3}}\right) \\
& \dot{x}_{4}=f_{4}\left(x_{3}, x_{4}, x_{5}, x_{2 \tau_{4}}, x_{3 \tau_{4}}, x_{4 \tau_{4}}\right) \\
& \dot{x}_{5}=f_{5}\left(x_{4}, x_{5}, x_{4 \tau_{5}}, x_{5 \tau_{5}}\right)
\end{aligned}
$$


where

$$
\begin{aligned}
f_{1}= & -\gamma_{1 h} x_{1}-\left(\eta_{1 h}+\eta_{2 h}\right) k_{h}\left(x_{2}+x_{4}\right) x_{1}-\left(1-\eta_{1 h}-\eta_{2 h}\right) \beta_{h}\left(x_{1}+x_{3}\right) x_{1}+ \\
& +2 e^{-\gamma_{1 h} \tau_{1}}\left(1-\eta_{1 h}-\eta_{2 h}\right) \beta_{h}\left(x_{1 \tau_{1}}+x_{3 \tau_{1}}\right) x_{1 \tau_{1}}+\eta_{1 h} e^{-\gamma_{1 h} \tau_{1}} k_{h}\left(x_{2 \tau_{1}}+x_{4 \tau_{1}}\right) x_{1 \tau_{1}} \\
f_{2}= & -\gamma_{2 h} x_{2}+A_{h}\left(2 \eta_{2 h}+\eta_{1 h}\right) k_{h}\left(x_{2 \tau_{2}}+x_{4 \tau_{2}}\right) x_{1 \tau_{2}} \\
f_{3}= & -\gamma_{1 l} x_{3}-\left(\eta_{1 l}+\eta_{2 l}\right) k_{l}\left(x_{2}+x_{4}\right) x_{3}-\left(1-\eta_{1 l}-\eta_{2 l}\right) \beta_{l}\left(x_{1}+x_{3}\right) x_{3}+ \\
& +2 e^{-\gamma_{1 l} \tau_{3}}\left(1-\eta_{1 l}-\eta_{2 l}\right) \beta_{l}\left(x_{1 \tau_{3}}+x_{3 \tau_{3}}\right) x_{3 \tau_{3}}+ \\
& +\eta_{1 l} e^{-\gamma_{1 l} \tau_{3}} k_{l}\left(x_{2 \tau_{3}}+x_{4 \tau_{3}}\right) x_{3 \tau_{3}}-b_{1} x_{3} x_{5} l_{1}\left(x_{3}+x_{4}\right) \\
f_{4}= & -\gamma_{2 l} x_{4}+A_{l}\left(2 \eta_{2 l}+\eta_{1 l}\right) k_{l}\left(x_{2 \tau_{4}}+x_{4 \tau_{4}}\right) x_{3 \tau_{4}}-b_{2} x_{4} x_{5} l_{1}\left(x_{3}+x_{4}\right) \\
f_{5}= & a_{1}-a_{2} x_{5}-a_{3} x_{5} l_{2}\left(x_{4}\right)+2^{n_{1}} a_{4} x_{5 \tau_{5}} l_{2}\left(x_{4 \tau_{5}}\right)
\end{aligned}
$$

We denote $X_{\tau}=X(t-\tau)$, where $X=\left(x_{1}, x_{2}, x_{3}, x_{4}, x_{5}\right)$. The history is introduced through:

$$
X(\theta)=\varphi(\theta), \theta \in\left[-\tau_{\max }, 0\right], \tau_{\max }=\max \left(\tau_{1}, \tau_{2}, \tau_{3}, \tau_{4}, \tau_{5}\right) .
$$

Two feedback rates are considered in the model: the rate of self-renewal $\beta(x)=\beta_{0} \frac{\theta_{1}^{m}}{\theta_{1}^{m}+x^{m}}$ and the rate of differentiation (through symmetric or asymmetric division) $k(x)=k_{0} \frac{\theta_{2}^{n}}{\theta_{2}^{n}+x^{n}}$. Because self-renewal is activated by signals from stem cell population and differentiation is activated by signals from mature cell population, it is assumed that $\beta$ depends on the sum of stem cell populations $\left(x_{1}+x_{3}\right)$, while $k$ depends on the sum of mature cell population $\left(x_{2}+x_{4}\right)$. In this way, competition between healthy and CML cell populations is considered here (see also [33]).

In this model, $\gamma_{1}$ and $\gamma_{2}$ are mortality rates, $\beta_{0}$ and $k_{0}$, the maximal rates of self-renewal, respectively of asymmetric division or differentiation into leukocyte line, $\theta_{1}$ and $\theta_{2}$ the values for which $\beta$, respectively $k$ attains half of their maximum value, $A$ an amplification factor of mature cells due to differentiation, $m$ is the parameter controlling the sensitivity of the rate $\beta$ to changes in the size of stem populations in $G_{0}$ and $n$ is the parameter controlling the sensitivity of the rate $k$ to changes in the size of mature population.

The fifth equation of the system models the anti-leukemia T cell immune response. We assume that after encountering a leukemic cell, a T cell has two alternatives: either inhibits the leukemic cell and activates a feedback function to stimulate the production of new T cells, or it is inhibited itself by the leukemic cell.

The interaction between T cells and CML cells was modeled through the functions $l_{1}(y)=\frac{1}{b_{3}+y}$ and $l_{2}(y)=\frac{y}{b_{4}+y^{2}}$. Hence, the last terms of the third and fourth equations stand for the inhibition of leukemic cells by anti-leukemia $\mathrm{T}$ cells. The function $l_{1}(y)$ was chosen starting from the assumption that the inhibition of the leukemic cell populations by the immune cells increases with the number of CML cells up to a certain level and then reaches a maximal value of inhibition. A further increase in the leukemic population will not modify this value.

As CML cell population stimulates anti-leukemia immune response only if leukemia cell population has values in a certain range, called "the optimal load zone" (see [21]) and inhibits the immune response if it exceeds a certain threshold value, to model the influence of CML cell population on $\mathrm{T}$ cell evolution, the feedback function $l_{2}(y)$ was chosen. Consequently, the third and the fourth terms of the fifth equation represents the rate at which naive T cells leave and re-enter the effector state after finishing the minimal developmental program of $n_{1}$ cell divisions, due to antigen stimulation. These terms reflect the process of production of T cells influenced by the CML cells.

The following parameters are related to the evolution of $\mathrm{T}$ cell population: $b_{1}$ represents the loss of leukemic stem cells due to cytotoxic T cells; $b_{2}$ is the loss of mature leukemic leukocytes due to cytotoxic T cells; $b_{3}$ represents a standard half-saturation in a Michaelis-Menten law $l_{1}(y) ; b_{4}$ is a standard half-saturation in a feedback function $l_{2}(y)$; $a_{1}$ represents the anti-leukemia T-cell natural supply rate; $a_{2}$ represents the anti-leukemia T-cell death rate; $a_{3}$ is the probability that a T cell survives the encounter with a leukemia cell; $a_{4}$ represents the coefficient of influence of the regulatory process; $n_{1}$ is the number of antigen depending divisions.

System (1) has four possible types of equilibria with non-negative components, namely: $E_{0}=\left(0,0,0,0, x_{5}^{*}\right)$, $E_{1}=\left(x_{1}^{*}, x_{2}^{*}, 0,0, x_{5}^{*}\right), E_{2}=\left(x_{1}^{* *}, x_{2}^{* *}, x_{3}^{* *}, x_{4}^{* *}, x_{5}^{* *}\right)$ and $E_{3}=\left(0,0, \tilde{x}_{3}, \tilde{x}_{4}, \tilde{x}_{5}\right)$, corresponding, from a medical point of view, to an extinction phase, to a healthy situation, to a chronic and respectively to an aggravated phase. Note that depending on the parameters values, the system can exhibit one or more equilibria of type $E_{3}\left(E_{3 . i}, i=1, p-\right.$ where the value of $\mathrm{p}$ depends on the biologically relevant range for cell populations).

The positive components of the equilibria $E_{1}$ satisfy:

$$
\gamma_{1 h}+\left[\eta_{1 h}\left(1-e^{-\gamma_{1 h} \tau_{1}}\right)+\eta_{2 h}\right] k_{h}\left(x_{2}^{*}\right)-\left(1-\eta_{1 h}-\eta_{2 h}\right)\left(2 e^{-\gamma_{1 h} \tau_{1}}-1\right) \beta_{h}\left(x_{1}^{*}\right)=0
$$




$$
-\gamma_{2 h} x_{2}^{*}+A_{h}\left(2 \eta_{2 h}+\eta_{1 h}\right) k_{h}\left(x_{2}^{*}\right) x_{1}^{*}=0 a_{1}-a_{2} x_{5}^{*}=0
$$

and the positive components of $E_{3}$ satisfy

$$
\begin{gathered}
\gamma_{1 l}+\left[\eta_{1 l}\left(1-e^{-\gamma_{1 l} \tau_{3}}\right)+\eta_{2 l}\right] k_{l}\left(\tilde{x}_{4}\right)-\left(1-\eta_{1 l}-\eta_{2 l}\right)\left(2 e^{-\gamma_{11} \tau_{3}}-1\right) \beta_{l}\left(\tilde{x}_{3}\right)-\gamma_{1} \tilde{x}_{3} \tilde{x}_{5} l_{1}\left(\tilde{x}_{3}+\tilde{x}_{4}\right)=0 \\
-\gamma_{2 l} \tilde{x}_{4}+A_{l}\left(2 \eta_{2 l}+\eta_{1 l}\right) k_{l}\left(\tilde{x}_{4}\right) \tilde{x}_{3}-b_{2} \tilde{x}_{4} \tilde{x}_{5} l_{1}\left(\tilde{x}_{2}+\tilde{x}_{4}\right)=0 \\
a_{1}-a_{2} \tilde{x}_{5}-\tilde{x}_{5} l_{2}\left(\tilde{x}_{4}\right)\left(a_{3}+2^{n_{1}} a_{4}\right)=0
\end{gathered}
$$

For a detailed theoretical analysis of the stability properties of the equilibrium points, please see [8] and [4].

\section{THE FRACTIONAL-ORDER DDE MODEL FOR CML}

In general, three different definitions of fractional derivatives, which are in general non-equivalent, are widely used: the Grünwald-Letnikov derivative, the Riemann-Liouville derivative and the Caputo derivative (see for example [29]). The main advantage of the Caputo derivative is that it only requires initial conditions given in terms of integerorder derivatives, representing well-understood features of physical situations and thus making it more applicable to real world problems. Highly remarkable scientific books which provide the main theoretical tools for the qualitative analysis of fractional-order dynamical systems, and at the same time, show the interconnection as well as the contrast between classical differential equations and fractional differential equations, are [29], [20], [22].

In the following, let us give the definition of the Caputo derivative and introduce the fractional-order DDEs system for CML.

Definition 1 For a continuous function $f$, with $f \in L_{l o c}^{1}\left(\mathbb{R}^{+}\right)$, the Caputo fractional-order derivative of order $q \in(0,1)$ of $f$ is defined by

$$
{ }^{c} D^{q} f(t)=\frac{1}{\Gamma(1-q)} \int_{0}^{t}(t-s)^{-q} f^{\prime}(s) d s,
$$

where the gamma function is defined, as usual, as $\Gamma(z)=\int_{0}^{\infty} e^{-t} t^{z-1} d t$.

Remark 2 When $q \rightarrow 1$, the fractional order derivative ${ }^{c} D^{q} f(t)$ converges to the integer-order derivative $f^{\prime}(t)$.

The fractional order DDE model for CML using the Caputo derivative is

$$
\begin{aligned}
{ }^{c} D^{q} x_{1} & =f_{1}\left(x_{1}, x_{2}, x_{3}, x_{4}, x_{1 \tau_{1}}, x_{2 \tau_{1}}, x_{3 \tau_{1}}, x_{4 \tau_{1}}\right) \\
{ }^{c} D^{q} x_{2} & =f_{2}\left(x_{2}, x_{1 \tau_{2}}, x_{2 \tau_{2}}, x_{4 \tau_{2}}\right) \\
{ }^{c} D^{q} x_{3} & =f_{3}\left(x_{1}, x_{2}, x_{3}, x_{4}, x_{5}, x_{1 \tau_{3}}, x_{2 \tau_{3}}, x_{3 \tau_{3}}, x_{4 \tau_{3}}\right) \\
{ }^{c} D^{q} x_{4} & =f_{4}\left(x_{3}, x_{4}, x_{5}, x_{2 \tau_{4}}, x_{3 \tau_{4}}, x_{4 \tau_{4}}\right) \\
{ }^{c} D^{q} x_{5} & =f_{5}\left(x_{4}, x_{5}, x_{4 \tau_{5}}, x_{5 \tau_{5}}\right) .
\end{aligned}
$$

Although the stationary points of the fractional-order DDEs system (4) are the same as in the case of the DDEs system (1), regarding their stability properties, things might be much different ([19]). Nevertheless, as for fractionalorder differential equations with time-delay only finite time stability results are known until now (see [23], [37]) and the general stability theory is not yet developed, in the following section, the dynamical behavior of the DDE model and its fractional counterpart will be analyzed and compared in view of the numerical results and simulations.

\section{NUMERICAL SIMULATIONS}

In this section, for the healthy cell populations we use the values of the parameters from [11], modifying some values of the CML cells parameters in view of medical evidences attesting that the values of leukemic parameters are increased or decreased in the case of leukemia. Accordingly, we consider for leukemic cells a smaller fraction of asymmetric division, a bigger fraction of self-renewal, lower rates of apoptosis for stem and mature cells, a bigger rate of self-renewal of stem cells, a bigger rate of asymmetric division or differentiation of stem cells and a bigger 
TABLE 1. Equilibrium points for the two configurations of parameters

\begin{tabular}{|c|c|c|}
\hline Equilibrium & Configuration 1 & Configuration 2 \\
\hline$E_{0}$ & $(0,0,0,0,13.04)$ & $(0,0,0,0,13.04)$ \\
\hline$E_{1}$ & $(0.13,4.46,0,0,13.04)$ & $(0.13,4.46,0,0,13.04)$ \\
\hline$E_{2}$ & $(0.09,0.23,0.06,27.76,25.76)$ & $(0.15,0.25,0.004,32.04,22.98)$ \\
\hline$E_{3.1}$ & $(0,0,0.06,27.61,25.88)$ & $(0,0,0.92,177.86,14.18)$ \\
\hline$E_{3.2}$ & $(0,0,0.56,62.46,16.88)$ & $(0,0,0.004,31.98,23.01)$ \\
\hline
\end{tabular}

amplification factor of mature cells. Furthermore, we considered that healthy cells have a propensity to asymmetric division, while CML cells have a predilection to self renewal ([35]). Obviously, as these values should be correlated with the features of the patient and his disease, there are many possible configurations of parameter space.

In all simulations, we choose the same set of values for the following parameters: $\beta_{0 h}=1.77 \cdot$ days $^{-1}, k_{0 h}=$ $0.1 \cdot$ days $^{-1}, \theta_{1 h}=\theta_{1 l}=0.5 \cdot 10^{6}$ cells $\mathrm{kg}^{-1}, \theta_{2 h}=\theta_{2 l}=36 \cdot 10^{8} \mathrm{cells}_{\mathrm{kg}}^{-1}, \mathrm{~m}=4, \mathrm{n}=3, \gamma_{1 h}=0.1 \cdot \mathrm{days}$, $\eta_{1 h}=0.7, \eta_{1 l}=0.1, \eta_{2 h}=0.1, \eta_{2 l}=0.7, \gamma_{2 h}=2.4, A_{h}=829, b_{1}=0.3, b_{2}=0.6, b_{3}=36, b_{4}=36, a_{1}=3$, $a_{2}=0.23, a_{3}=0.3, a_{4}=0.9, n_{1}=2, \tau_{1}=2.8, \tau_{2}=3.5, \tau_{3}=2.1, \tau_{4}=2.8, \tau_{5}=1.4$. For the rest of the parameters, in the following simulations, we consider two configurations of parameters describing two different forms of CML: configuration 1, with $\beta_{0 l}=2, k_{0 l}=0.4, \gamma_{1 l}=0.04, \gamma_{2 l}=1.5, A_{l}=2764.8$ and configuration 2, corresponding to a more serious disease, with $\beta_{0 l}=2.27, k_{0 l}=0.8, \gamma_{1 l}=0.01, \gamma_{2 l}=0.15, A_{l}=5529.6$. The values of the equilibrium points for the two configurations are listed in the table below.

Because the aim of this study is to compare the dynamical behavior of the model's trajectories in the case of the DDEs model and the modified fractional counterpart, in the following, numerical results and simulations pertaining to the stability properties of equilibria of type $E_{1}, E_{2}$, and $E_{3}$ will be presented. The history used in examples is constant and is taken in a neighborhood of the non trivial steady state.
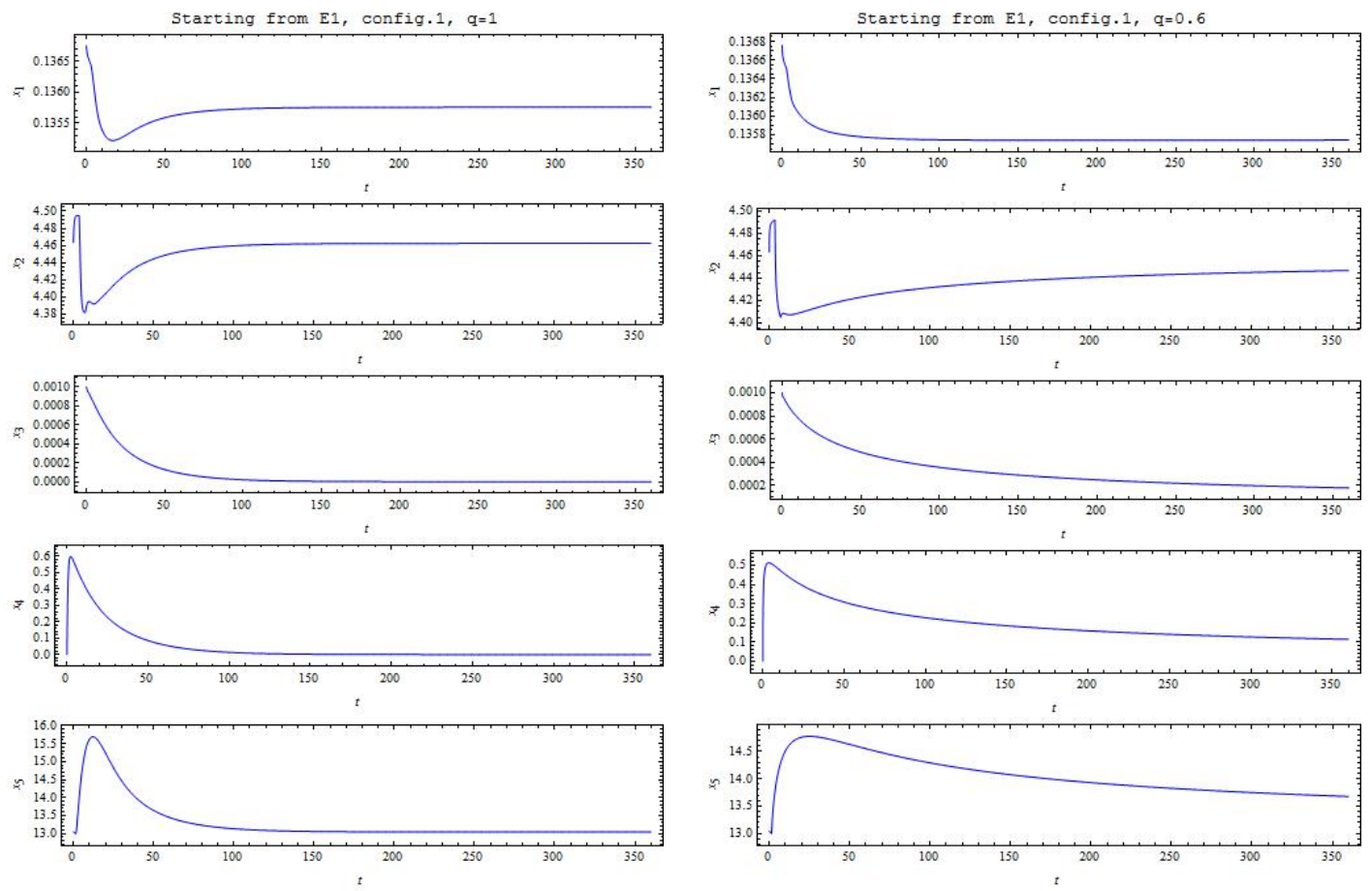

FIGURE 1. Configuration 1. Dynamics of hematopoietic populations starting from the neighborhood of $E_{1}$ for the integer-order DDEs model (left) and for the fractional-order DDEs model with $q=0.6$ (right). 

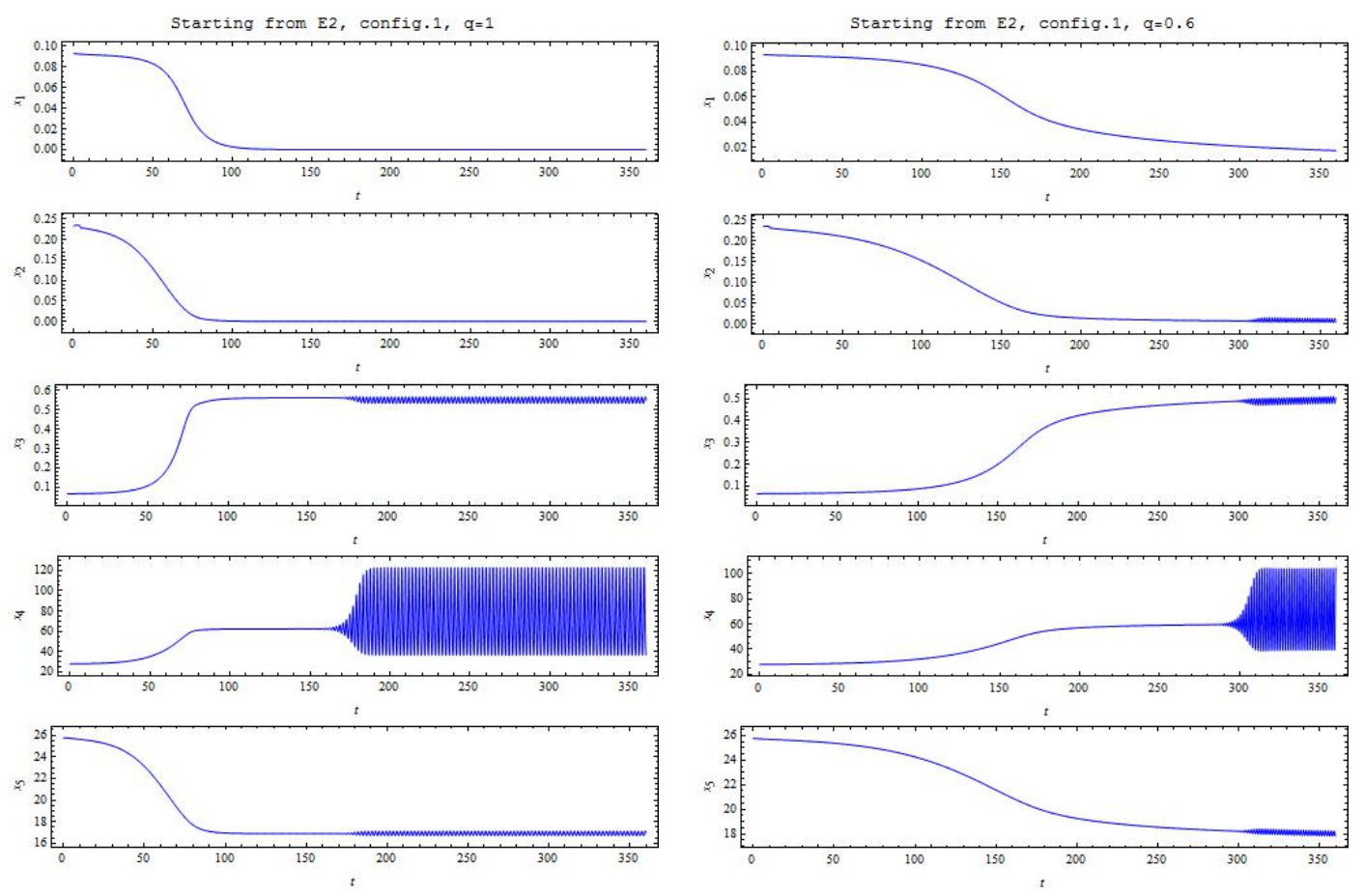

FIGURE 2. Configuration 1. Dynamics of hematopoietic populations starting from the neighborhood of $E_{2}$ for the integer-order DDEs model (left) and for the fractional-order DDEs model with $q=0.6$ (right).
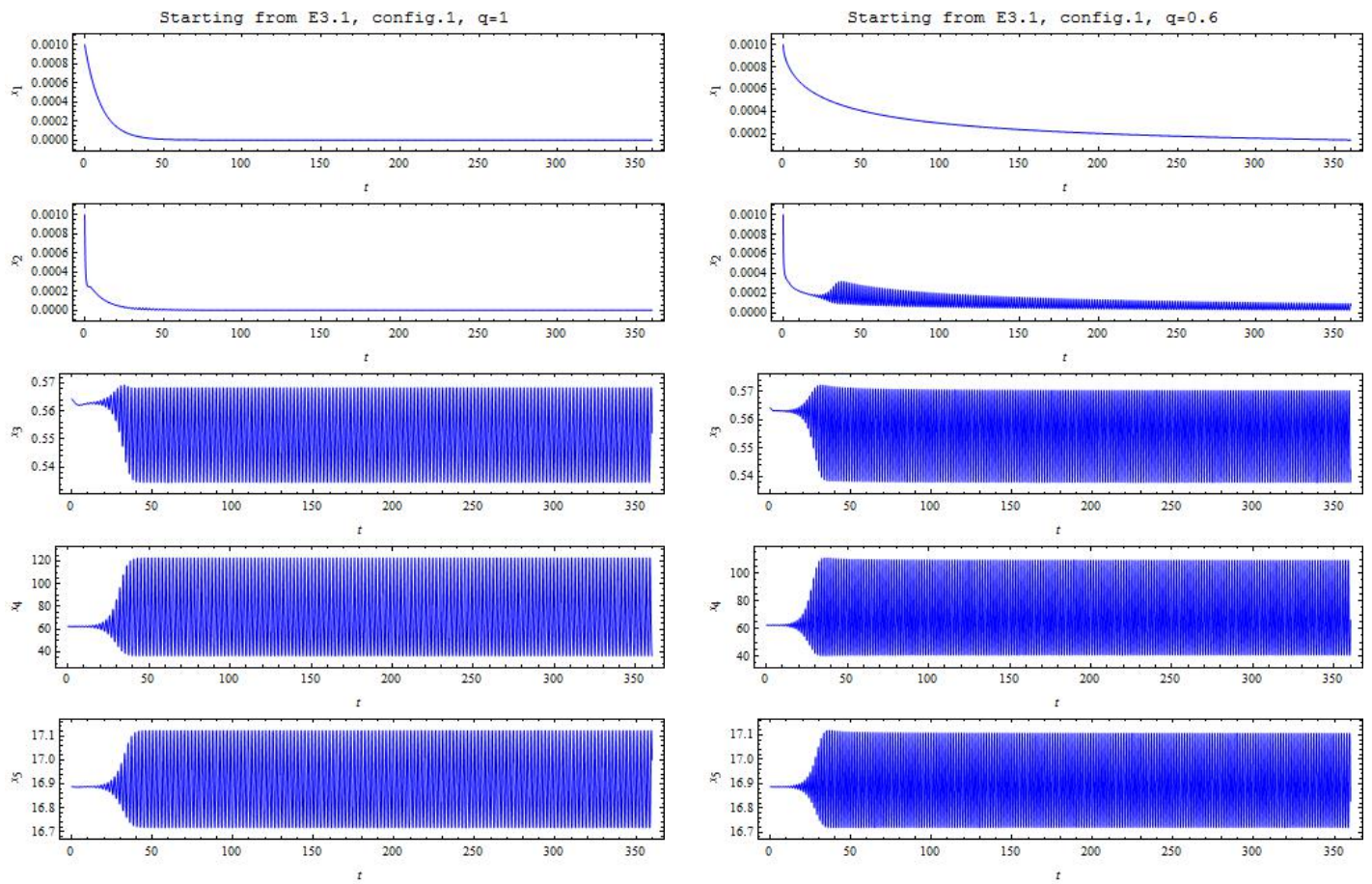

FIGURE 3. Configuration 1. Dynamics of hematopoietic populations starting from the neighborhood of $E_{3.1}$ for the integer-order DDEs model (left) and for the fractional-order DDEs model with $q=0.6$ (right). 

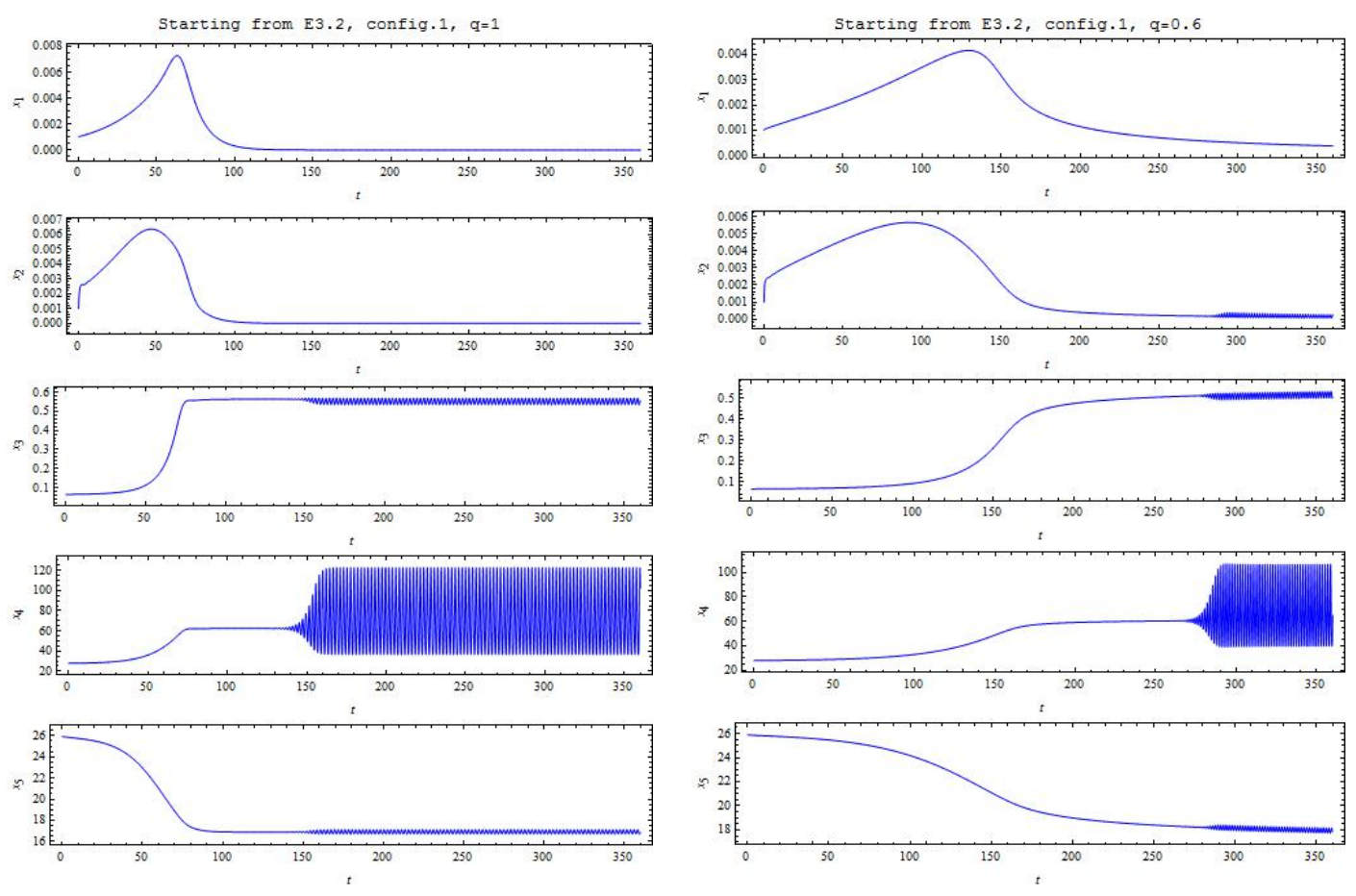

FIGURE 4. Configuration 1. Dynamics of hematopoietic populations starting from the neighborhood of $E_{3.2}$ for the integer-order DDEs model (left) and for the fractional-order DDEs model with $q=0.6$ (right).
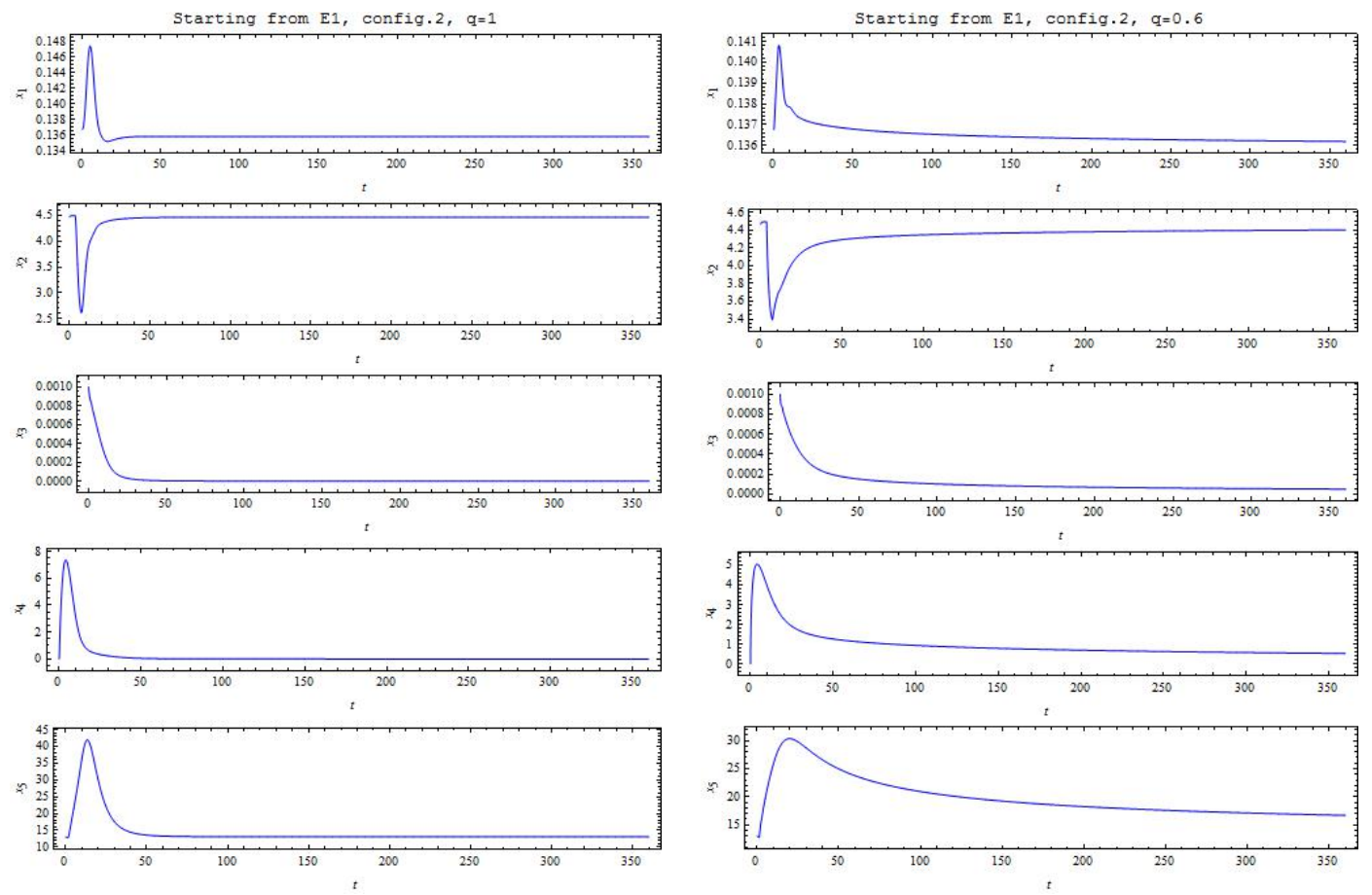

FIGURE 5. Configuration 2. Dynamics of hematopoietic populations starting from the neighborhood of $E_{1}$ for the integer-order DDEs model (left) and for the fractional-order DDEs model with $q=0.6$ (right). 

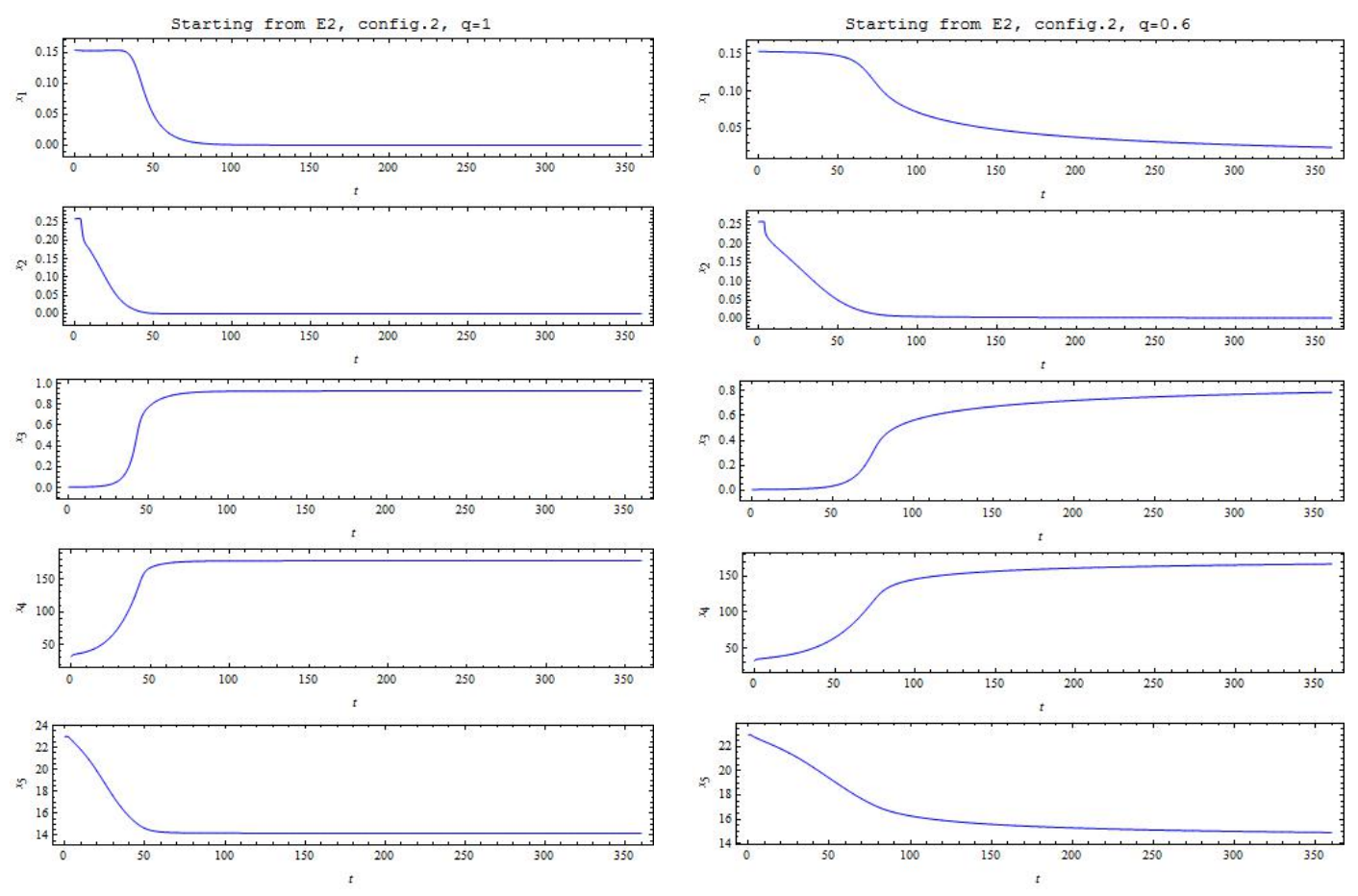

FIGURE 6. Configuration 2. Dynamics of hematopoietic populations starting from the neighborhood of $E_{2}$ for the integer-order DDEs model (left) and for the fractional-order DDEs model with $q=0.6$ (right).
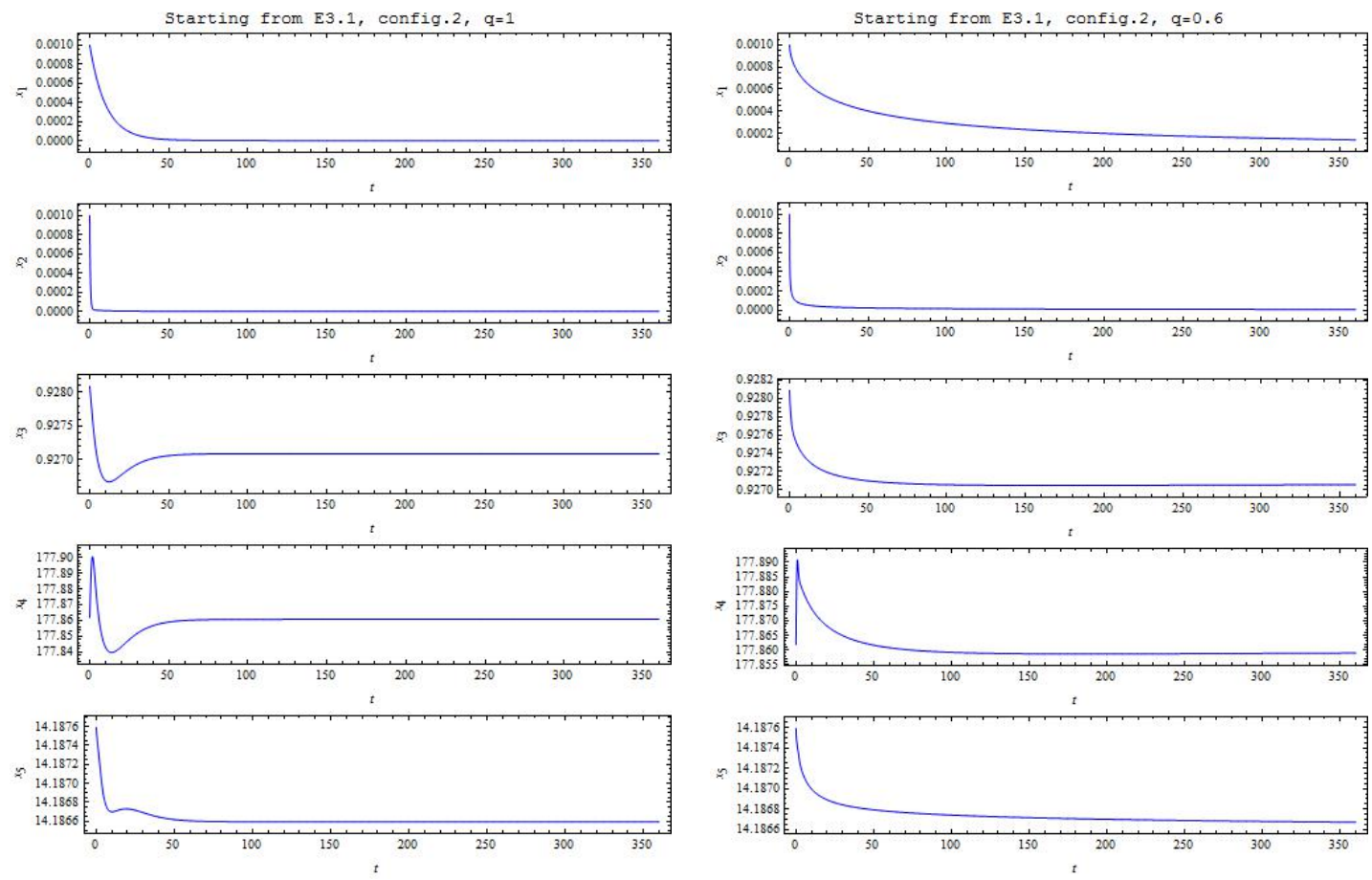

FIGURE 7. Configuration 2. Dynamics of hematopoietic populations starting from the neighborhood of $E_{3.1}$ for the integer-order DDEs model (left) and for the fractional-order DDEs model with $q=0.6$ (right). 

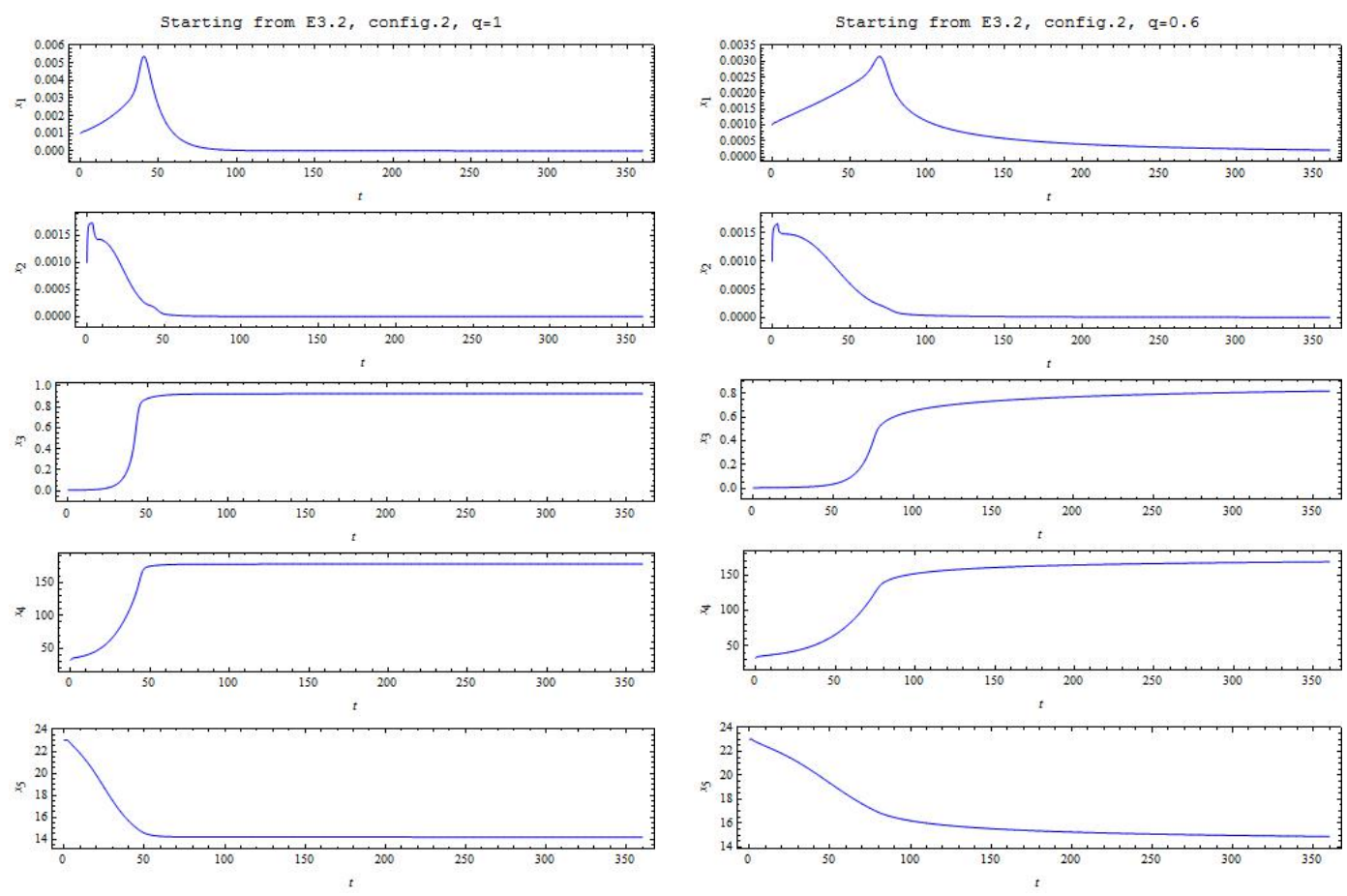

FIGURE 8. Configuration 2. Dynamics of hematopoietic populations starting from the neighborhood of $E_{3.2}$ for the integer-order DDEs model (left) and for the fractional-order DDEs model with $q=0.6$ (right).

\section{CONCLUDING REMARKS}

For both configurations of parameters, we found that equilibrium $E_{1}$ is stable and as the fractional order $q$ decreases the evolution is slower; equilibrium $E_{2}$ is unstable, and as the fractional order decreases, one can notice that the time period needed for the amount of the leukemic populations to rise up to a certain level become longer $(2-3$ months for $q=1$ and $5-6$ months for $q=0.6$ in the case of the first configuration and around 1 months for $q=1$ and 3 months for $q=0.6$ for the second configuration). For the two configurations of parameters values, two steady states of type $E_{3}$ can coexist: the first one (denoted $E_{3.1}$ ) can be either stable or unstable; the second one (denoted $E_{3.2}$ ) is always unstable. Equilibrium $E_{3.1}$ is unstable for the first configuration and stable for the second one, but the time evolution is very similar in the DDEs model and its fractional counterpart; equilibrium $E_{3.2}$ is unstable in both configurations, it seems to be attracted by $E_{3.1}$ for the second configuration and as the fractional order decreases, a very similar behavior as in the case of $E_{2}$ is noticed.

Consequently, an important conclusion can be drawn from the numerical simulations, as medical evidences regarding CML attest that the disease typically begins in the chronic phase and progresses to an accelerated phase over the course of several years, not only in few months. Hence, the results above are important findings suggesting that the introduction of fractional derivative into the DDEs system with a small fractional order $q$, might bring a more accurate representation of the reality into the model and improve it.

\section{ACKNOWLEDGEMENTS}

This work was supported by grants of the Romanian National Authority for Scientific Research and Innovation, CNCS-UEFISCDI, project no. PN-II-ID-PCE-2011-3-0198 (D. Cândea) and project no. PN-II-RU-TE-2014-4-0270 (I.R. Rădulescu and E. Kaslik). 


\section{REFERENCES}

[1] M. Adimy, F. Crauste, C. Marquet, Asymptotic behavior and stability switch for a mature-immature model of cell differentiation. Nonlinear Analysis: Real World Applications, 11, No. 4, 2913-2929 (2010).

[2] M. Adimy, F. Crauste, Delay Differential Equations and Autonomous Oscillations in Hematopoietic Stem Cell Dynamics Modelling, Math. Model. Nat. Phen. 7(6): 1-22 (2012).

[3] E. Ahmed, A.M. Elsayed, H.A.A. El-Saka, Equilibrium points, stability and numerical solutions of fractional-order predator-prey and rabies models, J. Math. Anal. Appl., 325, 542-553 (2007).

[4] I. Badralexi, A. Halanay, I.R. Radulescu, A Lyapunov-Krasovskii functional for a complex system of delaydifferential equations, UPB Sci. Bull., Series A, Vol. 77 (2): 9-18 (2015).

[5] E. Beretta, Y. Kuang, Geometric stability switch criteria in delay differential systems with delay dependent parameters, SIAM J. Math. Anal.33, no.5, 1144-1165 (2002).

[6] S. Bernard, J. Bélair, M.C. Mackey, Oscillations in cyclical neutropenia: New evidence for origins based on mathematical modeling, J. Theor. Biol. (2003), 223, 283-298 (2003).

[7] S.N. Catlin, P. Guttorp, J.L. Abkowitz, The kinetics of clonal dominance in myeloproliferative disorders, Blood 106:2688-2692 (2005).

[8] D. Cândea, A. Halanay, I.R. Radulescu, Stability analysis of some equilibria in a time-delay model for cell dynamics in leukemia including the action of the immune system, Math.Eng. Sci. Aerosp (MESA) 7, 1, 1-25 (2016).

[9] C.I. Chen, H.T. Maecker, P.P. Lee, Development and dynamics of robust T-cell responses to CML under imatinib treatment, Blood 111, 5342-5349 (2008).

[10] C. Colijn, A.C. Fowler, M.C. Mackey, High frequency spikes in long period blood cell oscillations, J. Math. Biol. 53, no.4, 499-519 (2006).

[11] C. Colijn, M.C. Mackey, A mathematical model of hematopoiesis - I. Periodic chronic myelogenous leukemia, J. Theor. Biol. 237, 117-132 (2005).

[12] M.W.N. Deininger, J.M. Goldman, J.V. Melo, The molecular biology of chronic myeloid leukemia, Blood $96,3343-3356(2000)$.

[13] M. Delitala, T. Lorenzi, M. Melensi, A Structured Population Model of Competition Between Cancer Cells and T Cells Under Immunotherapy, Mathematical Models of Tumor-Immune System Dynamics 107, Springer Proceedings in Mathematics \& Statistics, Springer New York, 47-58 (2014).

[14] D. Dingli, F. Michor, Successful therapy must eradicate cancer stem cells. Stem Cells 24:2603-2610 (2006).

[15] S. Faderl, M. Talpaz, Z. Estrov, S. O’Brien, R. Kurzrock, H. Kantarjian, The biology of chronic myeloid leukemia. New Engl. J. Med. 341 (3), 164-172 (1999).

[16] J. Foo, M.W. Drummond, B. Clarkson, T. Holyoake, F. Michor, Eradication of chronic myeloid leukemia stem cells: A novel mathematical model predicts no therapeutic benefit of adding G-CSF to imatinib, PLOS Comput Biol 5(9):e1000503 (2009).

[17] A. Halanay, D. Cândea, I.R. Rădulescu, Stability analysis of equilibria in a delay differential equations model of CML including asymmetric division and treatment, Math. Comp. Simul. 110, 69-82, (2015).

[18] A. Halanay, D. Cândea, I.R. Rădulescu, Existence and Stability of Limit Cycles in a Two Delays Model of Hematopoietis Including Asymmetric Division, Math. Model. Nat. Phen. 9, no. 1, 58-78 (2014).

[19] E. Kaslik, S. Sivasundaram, Analytical and numerical methods for the stability analysis of linear fractional delay differential equations, J. Comput. Appl. Math. 236, 4027-4041 (2012).

[20] A. Kilbas, H. Srivastava, J. Trujillo, Theory and Applications of Fractional Differential Equations, Elsevier, 2006.

[21] P.S. Kim, P.P. Lee, D. Levy, Dynamics and potential impact of the immune response to chronic myelogenous leukemia, PLoS Comput Biol. 2008 Jun; 4(6): e1000095 (2008).

[22] V. Lakshmikantham, S. Leela, J. V. Devi, Theory of fractional dynamic systems, Cambridge Scientifc Publishers, 2009.

[23] M.P. Lazarevic, D.L. Debeljkovic, Robust finite time stability of nonlinear fractional order time delay systems, Int. J. Inform. Sys. Sci. 4, 301-315 (2008).

[24] A.L. MacLean, C. Celso, M.P.H. Stumpf, Population dynamics of normal and leukaemia stem cells in the haematopoietic stem cell niche show distinct regimes where leukaemia will be controlled. J R Soc Interface 10(81):20120968 (2013). 
[25] A.L. MacLean, S. Filippi, M.P.H. Stumpf, The ecology in the hematopoietic stem cell niche determines the clinical outcome in chronic myeloid leukemia. Proceedings of the National Academy of Sciences of the United States of America, 111(10):3882-3888 (2014).

[26] S. Marley, M. Gordon, Chronic myeloid leukemia:stem cell derived but progenitor cell driven, Clinical Sciences 109, 13-25, 2006.

[27] H. Moore, N.K. Li, A mathematical model for chronic myelogenous leukemia (CML) and T cell interaction. J. Theor. Biol. 225: pp. 513-523 (2004).

[28] B. Neiman. A mathematical model of chronic myelogenous leukaemia. Master's thesis University College, Oxford University, (2002).

[29] I. Podlubny, Fractional differential equations, Academic Press, 1999.

[30] L. Pujo-Menjouet, M.C. Mackey, Contribution to the study of periodic chronic myelogenous leukemia,C.R.Biol.327(3), 235-244 (2004).

[31] I. Roeder, M. Horn, I. Glauche, A. Hochhaus, M.C. Mueller, M. Loeffler, Dynamic modeling of imatinibtreated chronic myeloid leukemia: functional insights and clinical implications, Nat Med 12:1181-1184 (2006).

[32] I.R. Rădulescu, D. Cândea, A. Halanay, A control delay differential equations model of evolution of normal and leukemic cell populations under treatment, IFIP Advances in Information and Communication Technology, Vol.443, 257-266, Springer (2014).

[33] I.R. Rădulescu, D. Cândea, A. Halanay, A study on stability and medical implications for a complex delay model for CML with cell competition and treatment, J. Theor. Biol., 363:pp. 30-40, ISSN: 0022-5193, DOI information: 10.1016/j.jtbi.2014.08.009 (2014).

[34] C. Riether, C.M. Schurch, A.F. Ochsenbein, Regulation of hematopoietic and leukemic stem cells by the immune system, Cell Death and Differentiation 22, 187-198 (2015).

[35] C. Tomasetti, D. Levy, Role of symmetric and asymmetric division of stem cells in developing drug resistance, Proceedings of the National Academy of Sciences 107 (39), 16766-16771 (2010).

[36] S.L. Topalian, G.J. Weiner, D.M. Pardoll, Cancer Immunotherapy Comes of Age. J Clin Oncol 29: pp. 48284836 (2011).

[37] X. Zhang, Some results of linear fractional order time-delay systems, Appl. Math. Comp. 197, 407-411 (2008). 